\title{
The Mushroom neutron spectrometer
}

\author{
R.I. Bewley
}

\section{Published version information}

Citation: R Bewley. 'The Mushroom neutron spectrometer.' Nucl Instrum Meth A, vol. 998 (2021): 165077.

DOI: $\underline{10.1016 / \text { j.nima.2021.165077 }}$

(C)2021. This manuscript version is made available under the CC-BY-NC-ND 4.0 Licence.

This version is made available in accordance with publisher policies. Please cite only the published version using the reference above. This is the citation assigned by the publisher at the time of issuing the AAM. Please check the publisher's website for any updates. 


\title{
R.I. Bewley
}

Isis Facility, Rutherford Appleton Laboratory, Chilton, Didcot, OX11 0QX, Oxfordshire, UK

\begin{abstract}
This paper presents a concept for a new type of in-direct time of flight cold neutron spectrometer called Mushroom. Mushroom has a unique pyrolytic graphite (PG) analyser/position sensitive detector geometry enabling a massive $2 \pi$ steradians of continuous position sensitive detector coverage, emulating some of its direct geometry counterparts such as IN5 or LET. It has many advantages over its direct geometry cousins though, being much more compact and much less expensive to build, but its biggest advantage is the order of magnitude larger count rates for the same resolution. It is envisaged it will be used to map out the 4 dimensional $\mathrm{S}(\mathrm{Q}, \omega)$ of single crystals in a matter of hours rather than days. Its design is aimed at minimising background and also uniquely allows easy selection of analyser reflection order PG002 or PG004. This paper presents details of the design along with full simulations of its potential performance using ray tracing software.
\end{abstract}

\section{Introduction}

The cold neutron direct-geometry (see figure 1 for meaning) time-of-flight (TOF) spectrometer is seen as an essential neutron instrument existing at nearly every neutron facility (CNCS at SNS (1) , IN5 at ILL (2), LET at ISIS (3), DCS at NIST (4), TOFTOF at FRM-II (5), AMATERAS at J-PARC (6)). One of the big advantages the Direct Geometry Spectrometer (DGS) is the ability to have a very large virtually continuous detector array, both in and out of plane often covering nearly $\pi$ steradians of solid angle. This enables large swathes of $S(Q, \omega)$ space to be measured in one shot. A fairly recent capability of these instruments thanks to the development of software such as $\operatorname{HORACE}(7)$, is to map out the complete $S(Q, \omega)$ space for single crystals samples (at least for the latest generation using position sensitive detectors). This is achieved by making many measurements at different rotation angles of the crystal and then combining all these measurements into a single $S(Q, \omega)$ using the HORACE software. A typical experiment would make 90 measurements with the sample rotated by 1 degree around a vertical axis for each measurement. The resultant $S(Q, \omega)$ file (typically around $150 \mathrm{~Gb}$ ) contains all the inelastic processes within the Brillouin zone and one can make 2 dimensional slices or 1 dimensional cuts in any direction from this data. There is increasing demand to do such measurements and instruments such as LET (3) and MERLIN (8) at the ISIS facility now spend the majority of beam time mapping $S(Q, \omega)$ for single crystals. However such measurements are very time consuming, typically taking a day or two to do a single scan, so it is not well suited for parametric studies. 
Another issue with the DGS stems from the fact that energy resolution is predominantly dependent on the sample to detector distance (9). Thus to achieve a reasonable resolution the instruments are very large with massive detector tanks and corresponding ${ }^{3} \mathrm{He}$ detector areas. For example the LET instrument has $40 \mathrm{~m}^{2}$ of ${ }^{3} \mathrm{He}$ position sensitive detectors set 3.5 $\mathrm{m}$ away from the sample. Not only do they take up large areas of real estate which can be problematic but since 2009 the cost of ${ }^{3} \mathrm{He}$ has sky rocketed (10) making them prohibitively expensive. Even if one could get the money, simply getting hold of such large volumes of ${ }^{3} \mathrm{He}$ is very difficult these days. This has been dubbed the 'Helium-3 crisis' and because of this there has been a concerted effort to find new detector technologies, in particular ${ }^{10} \mathrm{~B}$ thin film technologies (11) which will be used for two new DGS at the ESS (12), called CSPEC (13) and T-REX (14).

All of these DGS issues are circumvented with the new instrument concept presented in this paper called the Mushroom shown in figure 2. The Mushroom is an In-direct Geometry Spectrometer (IGS) (see figure 1 for meaning) utilising a large mushroom shaped pyrolytic graphite (PG) analyser scattering to a relatively small position sensitive detector array. Mushroom is designed to emulate a DGS like IN5 or LET in that it can detect neutrons scattered over a large solid angle both in and out of plane with continuous position sensitive coverage. It should be emphasised that in this aspect the Mushroom is very different from multi analyser systems being used on triple axis instruments such as flatcone (15) (16) or the new PUMA type multi analyser (17). These systems utilise multiple individual analysers in a single scattering plane and cover a relatively small solid angle.

It will be shown that the Mushroom is very compact and cheap compared to its DGS counterpart and in particular that it has an order of magnitude higher count rate for the same energy resolution. This potentially means that a full $S(Q, \omega)$ scan could be done in hours rather than days opening up the possibility of parametric studies and measurements on smaller samples.

The rest of this paper gives details and performance of Mushroom including full Monte Carlo simulations using the ray tracing software McStas (18). 


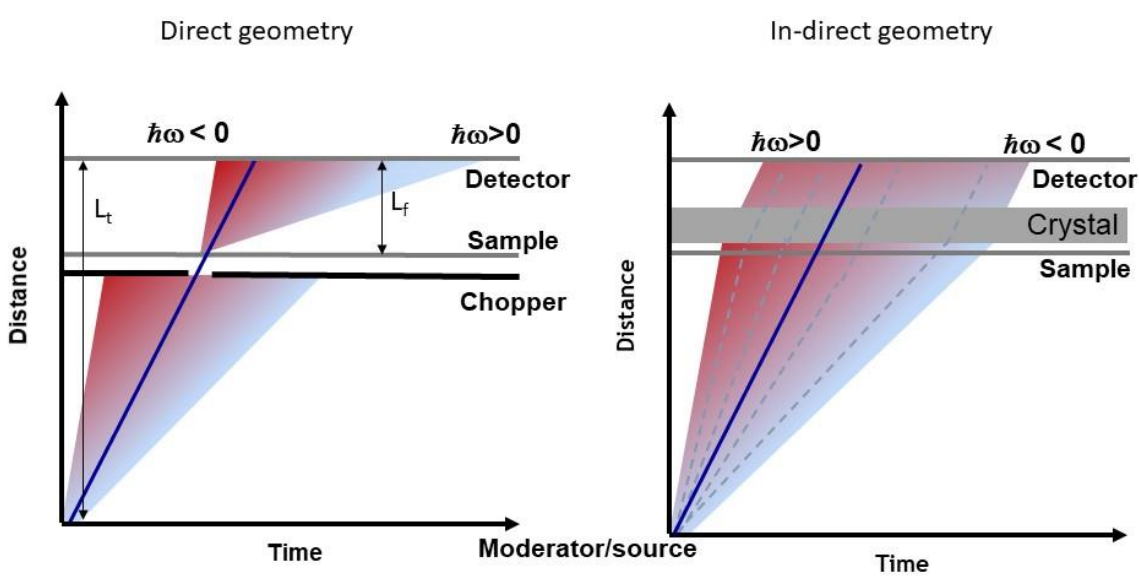

Figure 1 Distance time diagrams showing direct geometry (left) with monochromatic incident neutron beam on sample (usually from mechanical choppers) and a spread of final energies on detector . In-direct geometry (right) with white beam on sample but monochromatic final energy neutrons on detectors determined by crystal analyser. Both techniques use time of flight (TOF) to determine energy transfer to sample.

\section{The Mushroom instrument description}

The interesting and unique part of the Mushroom spectrometer is the analyser/detector geometry. The primary part of the spectrometer such as guides and choppers (delivering neutrons to the sample) are standard and details are not presented in this paper as they are not important to the Mushroom concept. In the following description the parameters are just stated, the justification for them come later in the paper. Figure 2 shows the Mushroom instrument with two large PG analysers, one either side of the sample. Each PG analyser is made from individual flat PG crystals measuring $1 \times 1 \mathrm{~cm}^{2}$ with a mosaic spread, $\mu$. Two values $\mu$ are studied in this paper, either $\mu=0.5^{\circ}$ or $\mu=0.8^{\circ}$, both values readily available commercially. The analysers cover a continuous range of scattering angles $2 \theta=10^{\circ}-170^{\circ}$ horizontally and $\phi=-5^{\circ}-50^{\circ}$ vertically around the sample. Providing enough space for sample environment equipment limits the maximum vertical value of $\phi$ to around $50^{\circ}$, this allows for a standard $40 \mathrm{~cm}$ diameter 'ISIS' flange.

Most importantly every point on the analyser will scatter to a unique point on the position sensitive detector which forms a horizontal sheet $90 \mathrm{~cm}$ below the sample position. The detectors are position sensitive ${ }^{3} \mathrm{He}$ tubes, $1.2 \mathrm{~m}$ long and $1 \mathrm{~cm}$ diameter. This simple arrangement allows for the first time a massive $2 \pi$ steradians of continuous in and out of plane detector coverage in an IGS. 
Figure $2 \mathrm{~b}$ shows a vertical section through the sample, analyser and detector. This figure shows that all scattered neutrons from any vertical angle pass through a radial focal point 85 $\mathrm{cm}$ from the sample origin and $28 \mathrm{~cm}$ below. There is a $4 \mathrm{~cm}$ wide radial opening in the neutron shielding centred on this focal radius through which the neutrons pass. The neutrons then pass through a rotating velocity selector before reaching the detector. The velocity selector is a large disk ( $85 \mathrm{~cm}$ radius) spinning around a vertical axis. At the edge of the disk there are many flat neutron absorbing blades set at an angle of $14^{\circ}$ off vertical. When spinning at $30 \mathrm{~Hz}$ this velocity selector lets through only the PG002 order and at $60 \mathrm{~Hz}$ the PG004 is selected. Focusing all the neutrons through a small opening in the shielding and then passing through a velocity selector is a critical part of the design in reducing background and possible spurious signals as well as cleanly separating the different orders scattered from PG. More on this will be said later.

Initially one may think that the vertical loci of the analyser must be elliptical in shape as it focuses all neutrons from one point (the sample) to the focal point at the velocity selector. However, a truly elliptical shape is not desirable as it would cause a large variation in the analysed energy, $E_{f}$, and corresponding energy resolution in going from the lowest to highest vertical angle $\phi$. Instead, specially written software starts laying down crystals vertically one at a time starting with the highest angle at $\phi=50^{\circ}$ which is set at $50 \mathrm{~cm}$ from the sample. The angle of each crystal is adjusted such that it reflects neutrons to the focal point, but a crystal can vary its position along the line bisecting the sample to crystal and crystal to the focal point. Each crystal is allowed to move by up to $3 \mathrm{~mm}$ relative to the crystal before it in order to try and keep the analysed energy constant. So moving a sample further away reduces $E_{f}$ or moving it closer increases it. Using this method the spread of $E_{f}$ is kept much smaller ranging from around $2.5 \mathrm{mev}$ from the bottom of analyser $(\phi=-5)$ to $3.1 \mathrm{mev}$ at $\phi=50^{\circ}$ for the PG002 reflection.

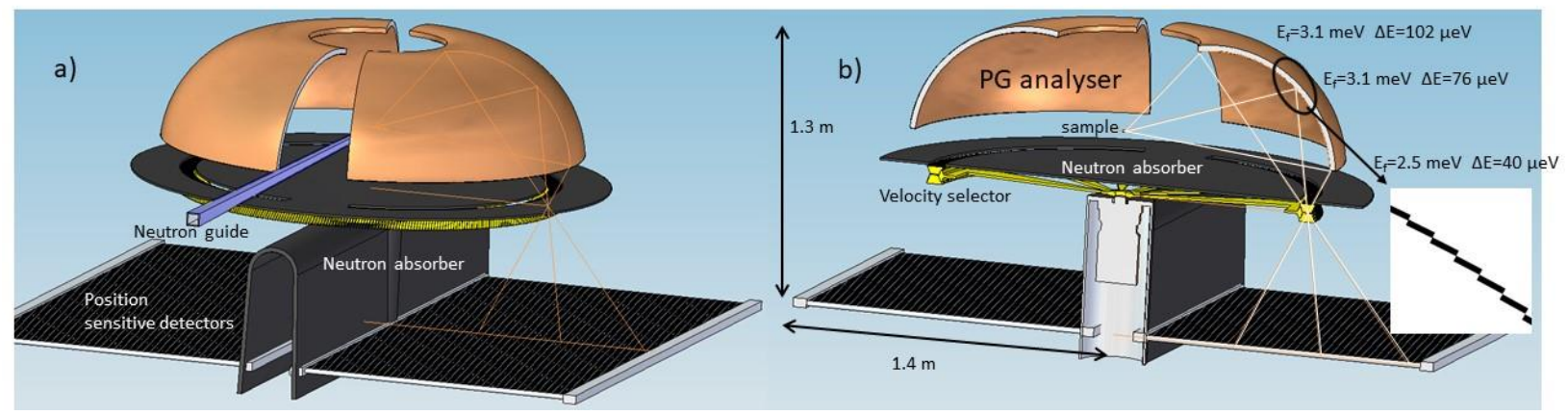

Figure 2 Engineering drawing of the Mushroom showing the essentials of the instrument. A vertical slice through the instrument b) shows some neutron ray paths from sample to analyser to detector along with the analysed energy $E_{f}$ and energy resolution of the elastic line $\Delta E$. The inset in b) shows a close up of how the crystals are stepped relative to each other. Each crystal is $10 \mathrm{~mm}$ long and $2 \mathrm{~mm}$ thick. 
Other possible analyser/detector geometries of the instrument have been studied but the one presented in figure 2 shows the most promise in terms of $Q$ resolution, background and simplicity.

\section{Performance and instrument simulations}

In the following the simulated performance of the energy resolution, $Q$ resolution and count rate of the Mushroom instrument is presented, along with a discussion of backgrounds. Full simulations were performed using the ray tracing software McStas (18). A moderator to sample distance of $25 \mathrm{~m}$ was chosen to be the same as the DGS LET (3) at ISIS to which comparisons are made. The sample size chosen for the Mushroom is $1 \times 1 \times 1 \mathrm{~cm}^{3}$ matching the $1 \times 1 \mathrm{~cm}^{2}$ size of the individual PG crystals and similarly the detector pixels were chosen to be $1 \times 1 \mathrm{~cm}^{2}$ in size. There was no component in McStas for the large Mushroom analyser so a new one was written which was a modification to the existing 'Monochromator_curved.comp' (19). The McStas simulations were output in a standard nexus file format which can be directly loaded into the software Mantid (20) (21), which is used by many neutron facilities for data reduction and analysis. Within Mantid a file is uploaded giving the $E_{f}$ and distance from sample to every detector pixel for the Mushroom instrument. One can use then utilise all the standard routines in Mantid to reduce and visualise data just as one does for the real spectrometers.

\subsection{Energy resolution}

Assuming the contributions are not correlated the energy resolution for an inverted TOF spectrometer like the Mushroom can be written as (22),

$$
\Delta E=2 \sqrt{E_{i}^{2}\left[\left(\frac{\Delta t_{i}}{t}\right)^{2}+\left(\frac{\Delta L}{L}\right)^{2}\right]+E_{f}^{2}\left[\left(\frac{\Delta t_{f}}{t}\right)^{2}+\left(\frac{\Delta d}{d}\right)^{2}+(\cot \theta \Delta \theta)^{2}\right]}
$$

where $\Delta t_{i}$ and $\Delta L$ are the time and distance uncertainties in the primary spectrometer. The time uncertainty comes mainly from the moderator pulse width which is around $110 \mu \mathrm{s}$ for the coupled hydrogen moderator at ISIS at $3 \mathrm{meV}$. $\Delta \mathrm{L}$ comes mainly from path uncertainties due to guide reflections and can be neglected (23). $L$ is the total length of instrument from the moderator to the detector ( $25 \mathrm{~m}$ from moderator to sample) and $t$ is the tof of the neutron. $\Delta t_{f}$ is any time uncertainty in the secondary spectrometer from things such as sample and detector size but is so small compared to the final term it can be ignored. $\Delta d / d=6 \times 10^{-4}$ (24) is the lattice spacing uncertainty of the PG crystals and $\theta$ is the Bragg angle of the analysed beam from the crystal which for the Mushroom varies from $55.0^{\circ}$ in plane (giving $\mathrm{E}_{\mathrm{f}}=2.7 \mathrm{meV}$ ) to $50.7^{\circ}$ at $\phi=50^{\circ}$ the largest vertical scattering angle (giving $\mathrm{E}_{\mathrm{f}}=3.1 \mathrm{mev}$ ). The 
uncertainty in the Bragg angle $\Delta \theta$ is from both the mosaic spread of the crystals, $\mu$, and the

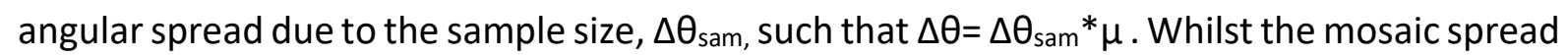
is fixed the value of $\Delta \theta_{\text {sam }}$ changes with vertical angle $\phi$ due to the variation in the sample to analyser distance $\left(95 \mathrm{~cm}\right.$ at $\phi=0^{\circ}$ to $50 \mathrm{~cm}$ at $\left.\phi=50^{\circ}\right)$. This is the reason for the worsening energy resolution with increasing $\phi$ shown in figure $2 \mathrm{~b}$. Figure 3 shows McStas simulations of the elastic line both in plane, $\phi=0$, and at a $\phi=45^{\circ}$ for a mosaic spread $\mu=0.5^{\circ}$ for the PG002 reflection giving full width half maximum (FWHM) resolutions of $46 \mu \mathrm{eV}$ and $97 \mu \mathrm{eV}$ respectively, very close to the expected resolutions of $52 \mu \mathrm{eV}$ and $97 \mu \mathrm{eV}$ using equation 1. The asymmetry in the elastic line shape shown in figure 3 is due to the moderator component which is very asymmetric for the coupled hydrogen moderator used in the simulations.

All the terms in equation 1 are important for high resolution backscattering spectrometers such as IRIS (25) or OSIRIS (26) where the final term in equation 1 vanishes as $\theta$ goes to $90^{\circ}$. The Mushroom is well away from backscattering and the final term dominates at the elastic line. However, at higher energy transfers the first term in equation 1 starts to dominate. Figure 4 shows the calculated energy resolution of the Mushroom versus energy transfer (Etran) for neutron energy loss.

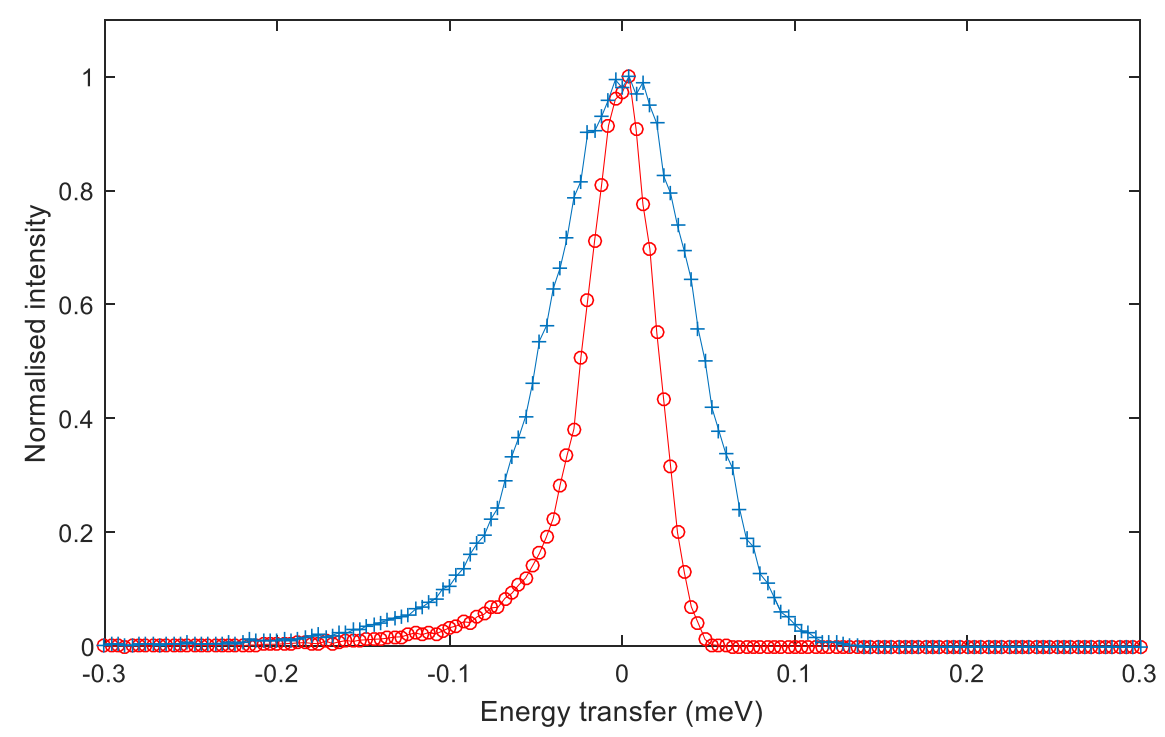

Figure 3. Simulated elastic resolution for PG002 of Mushroom with $\mu=0.5^{\circ}$. The maximum intensity has been normalised to 1 . The circles show data in plane $\left(\phi=0^{\circ}\right)$ with a $46 \mu \mathrm{eV}$ resolution. Crosses show $\phi=45^{\circ}$ with a resolution of $97 \mu \mathrm{eV}$. 

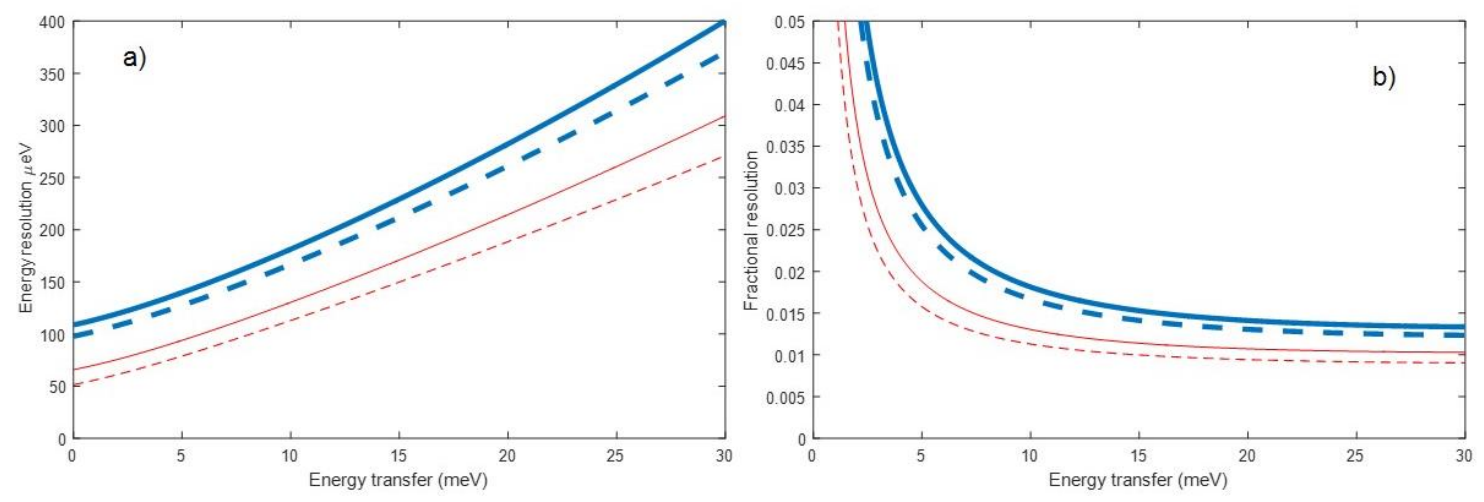

Figure 4 Calculated energy resolution a) and fractional energy resolution $\left.\Delta E / E_{\text {tran }} b\right)$. The solid lines represent $\mu=0.8^{\circ}$ and dashed $\mu=0.5^{\circ}$. Thick lines are at $\phi=45^{\circ}$ while thin lines are at $\phi=0^{\circ}$.

The energy resolution can be improved further at higher energy transfers by increasing the moderator to sample distance which reduces the first term in equation 1 . This will have little effect on the sample flux as a good modern guide should transport it with only small losses. So ideally one should increase $L$ to be as long as possible to fill the time frame, with the final length depending on the energy transfer range required and the repetition rate of the source. Figure $4 \mathrm{~b}$ shows that even at a relatively short distance of $25 \mathrm{~m}$ the Mushroom has an excellent energy resolution, with a fractional energy resolution $\Delta E / E_{\text {tran }} \approx 1 \%$ over much of its range.

\subsection{Q Resolution}

If the Mushroom is to fulfil its purpose of mapping out the 4D Brillouin zone for single crystals then it is crucial it has a reasonable $Q$ resolution. Unlike a DGS where the neutrons scatter directly from the sample to the detector, for the Mushroom they reach the detector via the $P G$ analyser which has a mosaic spread $\mu$. For this reason the $Q$ resolution of the Mushroom will not be as good as its DGS counterparts but with careful design respectable $Q$ resolutions can be obtained as will be demonstrated. One could simply choose crystals with a very low mosaic spread but this reduces the spectrometers detector count rate as the wavelength spread 'analysed' by the PG crystals is given by $\Delta \lambda_{P G}=\lambda_{P G} \cot \theta . \mu$. It's the usual case of resolution versus count rate. Also there is no point in making $\mu$ much smaller than $\Delta \theta_{\text {sam }}$ as this will then dominate the $Q$ resolution. Ideally one matches them such that $\mu=\Delta \theta_{\text {sam }}$ but for the Mushroom $\Delta \theta_{\text {sam }}$ varies with vertical scattering angle from $\approx 0.5^{0}$ in-plane to $\approx 1^{0}$ at $50^{\circ}$ scattering angle. In the simulations presented in this paper the values of $\mu$ are $0.5^{\circ}$ and $0.8^{\circ}$ as they are both commercially available and also approximately match $\Delta \theta_{\text {sam }}$.

The aim is to optimise the $Q$ resolution of the secondary spectrometer so for now contributions from the primary are neglected. In the McStas simulations presented this is achieved by using a perfectly collimated incident beam. The three components of the $Q$ resolution are the vertical component, $\Delta \mathrm{Q}_{y}$, the component along the incident beam, $\Delta \mathrm{Q}_{z}$, and the component perpendicular to the incident beam but in plane $\Delta \mathrm{Q}_{\mathrm{x}}$ (perpendicular to both 
$\Delta \mathrm{Q}_{\mathrm{y}}$ and $\Delta \mathrm{Q}_{\mathrm{z}}$ ). Each of these components is a function of both $\theta$ and $\phi$, thus giving many thousands of values in the highly pixelated detector. To simplify the optimisation all simulations were performed at $2 \theta=90^{\circ}$, representing a vertical strip in the middle of the analyser/detector bank. There are 4 parameters to optimise in this instrument geometry, the distance $R$ to the first crystal $\left(\phi=50^{\circ}\right)$, the value of $E_{f}$ from this crystal which defines the line on which the focal point, FP, will lie, the distance along this line to FP and finally the vertical distance the detectors sit below FP is $Y_{\text {det }}$. Specially written software scans through this 4 dimensional phase space numerically calculating values of $\Delta \mathrm{Q}_{y}(\phi), \Delta \mathrm{Q}_{z}(\phi)$ and $\Delta \mathrm{Q}_{x}(\phi)$ at every point along $\phi$. The maximum values $\Delta \mathrm{Q}_{y}(\phi)^{\max }, \Delta \mathrm{Q}_{z}(\phi)^{\max }$ and $\Delta \mathrm{Q}_{x}(\phi)^{\text {max }}$ are recorded at each point in the 4D phase space and the best set of parameters was chosen as the set which minimised the sum of the squares $\Delta Q_{\text {tot }^{2}}{ }^{2}\left(\Delta Q_{y}{ }^{\max }\right)^{2}+\left(\Delta Q_{x}{ }^{\max }\right)^{2}+\left(\Delta Q_{z}{ }^{\text {max }}\right)^{2}$. The software numerically calculating the three components by simply calculated the FWHM spread of the neutron beam at the detector as a function of $\phi$ for each PG crystal, incorporating the effects of mosaic spread, sample size and angle the beam hits the detector. The spread along the $z$ direction on the detector converts to $\Delta \mathrm{Q}_{z}$ while the spread along $\mathrm{x}$ direction converts to $\Delta \mathrm{Q}_{\mathrm{x}}$ and $\Delta \mathrm{Q}_{\mathrm{y}}$. Although rather crude in that it does not account for possible correlations, it is very fast and allows a rapid transit through the large phase space which would not be feasible in a reasonable time frame with simulations. Justification for the simple technique is shown in Figure 5 showing the numerical calculations for the optimised Mushroom along with full McStas simulations of these components. It can be seen there is good agreement between the two techniques. The McStas simulations used a virtual sample which creates perfect one dimension rods in Q space, either vertically or horizontally. These rods are broadened due to instrumental effects giving the $Q$ resolution. The data was reduced in Mantid (described earlier) and the width of the $Q$ rods was then fitted with a Gaussian function and the FWHM of this versus $\phi$ is presented in Figure 5.

It was found during the optimisation process that the $Q$ resolution asymptotically improves with $R$, which is to be expected as the effect of sample size gradually reduces with increasing $R$. However, the cost of the analyser will go as $R^{2}$ so in the end $R$ was fixed at $0.5 \mathrm{~m}$ giving a contribution to the $Q$ resolution less than that given by the guide divergence and a reasonable cost and size for the Mushroom analyser. This is in much the same way that the energy resolution improves with sample to detector distance in a DGS but cost and size limits the distance chosen. The optimisation program always keeps FP close to the PG analyser which reduces beam spreading from the $P G$ mosaic thus improving $Q$ resolution. At FP the vertical beams all converge and a detector here, $Y_{\text {det }}=0$, would have no $Q$ resolution. On increasing $\mathrm{Y}_{\text {det }}$ then $\Delta \mathrm{Q}_{\mathrm{x}}$ and $\Delta \mathrm{Q}_{\mathrm{y}}$ improve with the increased resolving power as the beams diverge away from FP. This also reaches an asymptotic limit due to the opposing effect of increased beam spread with increasing $Y_{\text {det. }}$. As $Y_{\text {det }}$ increases scattering from the smallest $\phi$ reaches the detector closer and closer to zero radius rapidly degrading $\Delta Q_{z}$ as can be seen in figure $5 . A$ reasonable balance between $\Delta Q_{z}$ increasing and $\Delta Q_{x}, \Delta Q_{y}$ decreasing with increasing $Y_{\text {det }}$ occurs when $Y_{\text {det }} \approx 90 \mathrm{~cm}$. Similarly the program optimised $E_{f}$ around $3 \mathrm{meV}$ which creates an analysed beam which on average is coming vertically down so hitting the flat horizontal detector as perpendicular as possible. The $Q$ resolution could in principle be further improved if the detectors were not flat but on circular loci around FP. Then the beam would always hit the detectors perpendicularly thus reducing beam spread and $\Delta \mathrm{Q}_{\mathrm{z}}$ would not degrade so 
much at low $\phi$. However, with the aim of trying to keep the instrument simple and reasonable we will stick to the simple flat detector array.

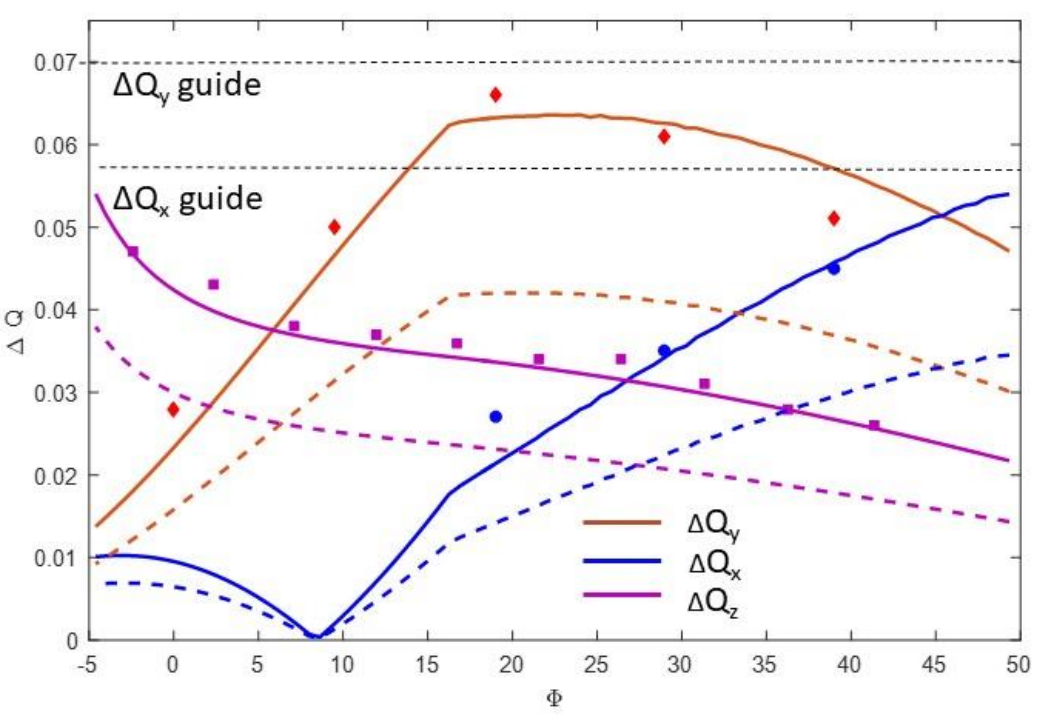

Figure 5. The three components of the Mushroom Q resolution at $2 \theta=90^{\circ}$ versus the vertical angle $\phi$. Solid lines represent the simple numerical calculations for $\mu=0.8^{\circ}$ and dashed lines represent $\mu=0.5^{\circ}$. The square markers represent the results of full simulations at $\mu=0.8^{\circ}$. The thin dashed lines at the top show the uncertainty in $\Delta \mathrm{Q}_{x}$ and $\Delta \mathrm{Q}_{\mathrm{y}}$ due to the incoming guide for $3 \mathrm{meV}$ neutrons.

To see how well the optimised version of the Mushroom instrument performs a full McStas simulation was done on a virtual crystal with a 2 dimensional (2D) spin wave in the $x-z$ plane (dispersion less along $\mathrm{y}$ ). The spin wave had a periodicity of $1 \AA^{-1}$ in both $\mathrm{x}$ and $\mathrm{z}$ directions and had a band maximum of $4 \mathrm{meV}$. It is not possible to do a direct simulation comparison with a DGS like LET as they are very different instruments but simulation parameters for LET were chosen be reasonable values for such an experiment. So an incident energy of $\mathrm{Ei}=5 \mathrm{meV}$ was picked, to just cover the $4 \mathrm{meV}$ band maximum, and the final monochromating chopper spun at $300 \mathrm{~Hz}$ (maximum) giving an energy resolution at the elastic line of about $100 \mu \mathrm{eV}$ compared to Mushrooms $46 \mu \mathrm{eV}$. The Mushroom was put on the end of the LET guide (choppers removed) which has a moderator to sample distance of $25 \mathrm{~m}$. The simulations involved a HORACE scan of the virtual 2D crystal through $90^{\circ}$ in $1^{\circ}$ steps and then the simulated data was reduced and analysed in exactly the same way we would with experimental data from the real instruments.

Figure 6 shows the results of the Mushroom and LET simulations in 3 dimensions, with the momentum transfer in plane, $Q_{a}$ and $Q_{b}$, and energy transfer. The simulations show that Mushroom performs well and gives similar results compared to LET. 

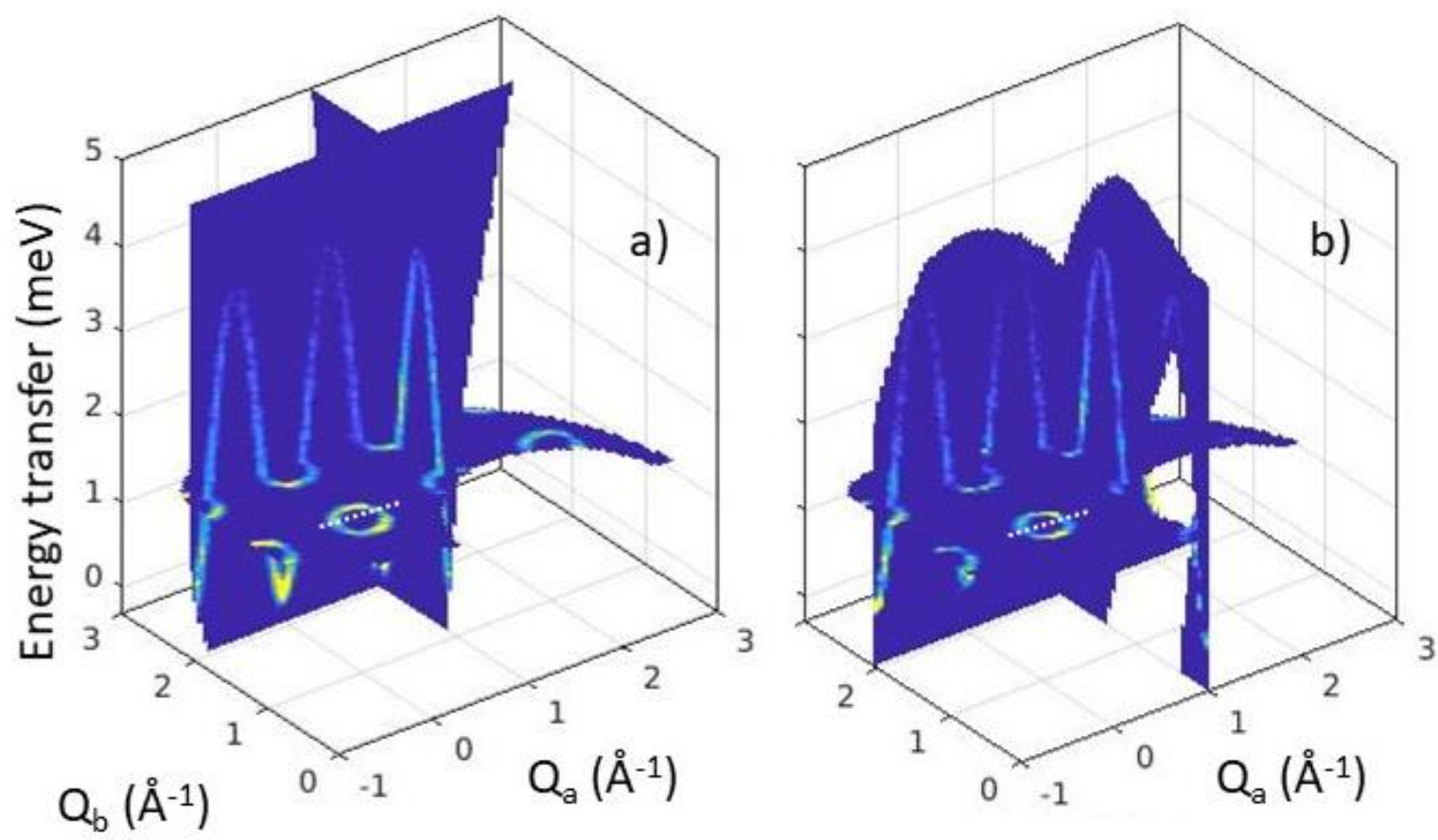

Figue 6. Full McStas simulations of $2 D$ spin waves in a crystal with a periodicity of $1 \AA^{-1}$ along $Q_{a}$ and $Q_{b}$ and a 4 meV band maximum. The simulations are for a) Mushroom and b) LET. The white dashed line shows the cut shown in figure 7.

A more detailed comparison is presented in figure 7 showing simulated data along an identical cut, indicated by the dashed line in figure 6 . The cut has a width of $0.1 \AA^{-1}$ along $Q_{b}$ and 0.1 $\mathrm{meV}$ in energy transfer. It can be seen that the $Q$ resolution of Mushroom is only slightly worse than LET. Like most inelastic spectrometers the LET guide has a large incident beam divergence to increase sample flux. At $3 \mathrm{meV}$ the incoming momentum uncertainty due to the incident divergence is $\Delta \mathrm{Q}_{\mathrm{x}}=0.058 \AA^{-1}$ and $\Delta \mathrm{Q}_{\mathrm{y}}=0.07 \AA^{-1}$ as shown by the dashed horizontal lines on figure 5 . These values are similar to the momentum uncertainties due to the Mushroom secondary spectrometer and therefore it is not surprising that the Mushroom $Q$ resolution is only slightly worse than LET. It can be seen in figure 7 that there is a slight discrepancy in the second peak position between LET and Mushroom. The Mushroom peak is in the correct position but the reason for the slight discrepancy in the LET peak position is not understood. 


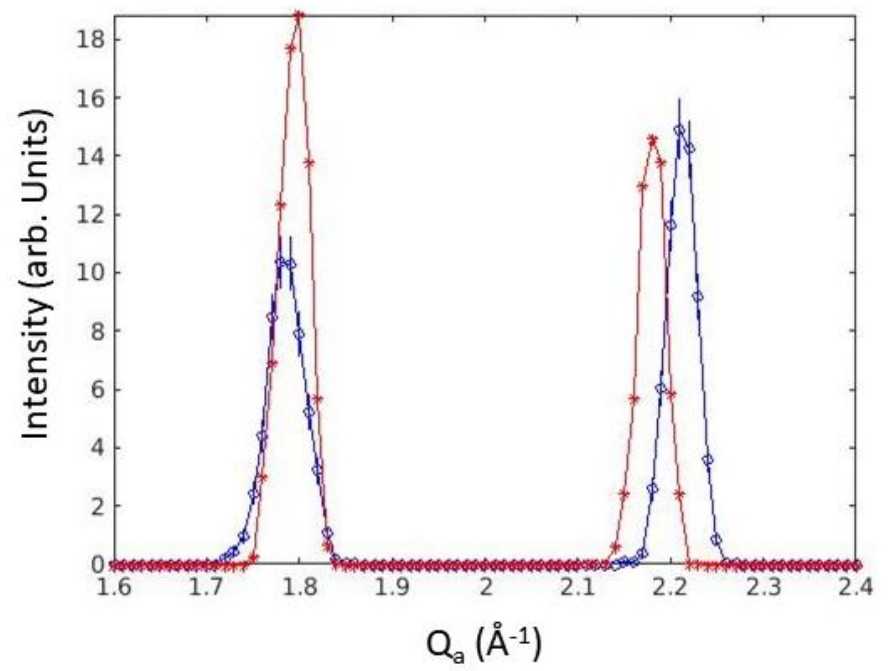

Figure 7. A cut through the simulation shown in figure 6 as indicated by the dashed line at $Q_{b}=1$ and energy transfer of $1.5 \mathrm{meV}$. Mushroom data is represented with the circles and stars LET. The intensity is arbitrary and has been scaled so they are roughly the same height.

\subsection{Count rate}

When comparing the performance of spectrometers the incident neutron flux on the sample is often given as one of the performance criteria. However, this is a useless measure when comparing Mushroom to a DGS with one having a monochromatic incident beam and the other a whitebeam. The important measure is the detector count rate for the same sample, resolution and solid angle. In this section it is shown that the Mushroom has much higher detector count rates than a DGS using the same neutron guide. This is because indirect geometry machines are much more efficient than direct geometry which stems from the fact that energy resolution of indirect instruments comes from the full instrument length whereas it's predominantly the much shorter length of the secondary spectrometer in DGS instruments.

A simple analytical argument is given below which is then backed with simulations. The energy resolution, $\Delta \mathrm{E}$, of an indirect and direct geometry instrument are given by equations 2 and 3 respectively.

$$
\left.\left.\frac{\Delta E}{E_{i}}=\frac{2 \Delta t^{i}}{t_{t}^{i}} 2\right) \quad \frac{\Delta E}{E_{f}}=\frac{2 \Delta t^{d}}{t_{f}^{d}} 3\right)
$$

The superscript ' $\mathrm{i}$ ' and ' $\mathrm{d}$ ' denotes in-direct and direct respectively. $\Delta \mathrm{t}^{\mathrm{i}}$ and $\Delta \mathrm{t}^{\mathrm{d}}$ is the total time spread/uncertainty at the detectors due to all the resolution components. $E_{i}$ and $E_{f}$ are the incident and final neutron energy and $t_{t}^{i}$ is the total time of flight (moderator to detector) and $t^{d}{ }_{f}$ is the time of flight from sample to detector. At the elastic line such that $E_{i}=E_{f}$ and for equal energy resolutions equation 2 and 3 combine to give

$$
\frac{\Delta t^{i}}{\Delta t^{d}}=\frac{t_{t}^{i}}{t_{f}^{d}}
$$


The Mushrooms energy resolution is dominated by the mosaic spread of the PG crystals (shown in section 3.1) which take a wavelength spread $\Delta \lambda^{i}$ from the scattered beam. One can express $\Delta \mathrm{t}^{i}$ in equation 4 ) in terms of $\Delta \lambda^{i}$ such that

$\Delta \mathrm{t}^{\mathrm{i}}=\mathrm{t}^{\mathrm{i}} \mathrm{t} \Delta \lambda^{\mathrm{i}} / \lambda$

5)

Similarly for a DGS spectrometer like LET one can express $\Delta \mathrm{t}^{\mathrm{d}}$ in equation 4 ) in terms of the wavelength spread $\Delta \lambda^{d}$ taken by the final monochromating chopper such that

$\Delta \mathrm{t}^{\mathrm{d}}=\mathrm{t}^{\mathrm{d}}{ }_{\mathrm{t}} \Delta \lambda^{\mathrm{d}} / \lambda$

This assumes that the resolution is dominated by the final chopper term which is usually the case although not at very high energy resolutions. Combining equations 4), 5) and 6) we get

$$
\left.\frac{\Delta \lambda^{i}}{\Delta \lambda^{d}}=\frac{t_{t}^{d}}{t_{f}^{d}}=\frac{L_{t}^{d}}{L_{f}^{d}} \quad 7\right)
$$

The ratio of the detector count rates for the indirect over the direct spectrometer is given by $\Delta \lambda^{i} / \Delta \lambda^{d}$ as this represents the wavelength spread taken from the moderator for each spectrometer for an equivalent energy resolution. $A s L_{t}^{d}>>L^{d}{ }_{f}$ we can immediately see that the indirect machine 'extracts' far more flux for the same resolution as the direct machine. Typical $L_{t}{ }_{t} / L^{d}{ }_{f} \approx 10$ for cold neutron spectrometers like LET, AMATERAS and CNCS. This does not take into account any losses due to reflectivity of the crystals but for the Mushroom this loss is small as PG crystals have a high reflectivity of $\approx 90 \%$.

To check this result simulations have been performed comparing the detector count rates for Mushroom and LET. In the simulations the Mushroom has again been put on the end of the LET neutron guide and both instruments used a $1 \times 1 \times 1 \mathrm{~cm}^{3}$ vanadium sample scattering to exactly the same solid angle of $5 \times 5^{\circ}$ and both had the same resolution at the elastic line of $48 \mu \mathrm{eV}$. To achieve this resolution on LET the incident energy was set to $\mathrm{Ei}=2.8 \mathrm{meV}$ which is the same as the final energy of the Mushroom (in plane) and the final chopper was run at $260 \mathrm{~Hz}$ with a $28 \mathrm{~mm}$ opening. This is an optimal setup for LET. Figure 8 shows the simulation of the elastic line for both instruments and demonstrates the same energy resolution. The intensities have been normalised to 1 for easy comparison. It can be seen that Mushroom has a 'tail' on the neutron energy gain side which was discussed in section 3.1 as being due to the asymmetric moderator component. The LET spectrometer has a 'tail cutting' chopper at the start of the instrument which eliminates this feature. In the simulations Mushroom had a count rate of 12.40 neutrons per second on the detector while LET had 1.52 neutrons per second. This means Mushroom had an increased count rate of 8x LET for exactly the same sample, resolution and solid angle. Using equation 7 where $L^{d}=28.5 \mathrm{~m}$ and $L^{d}{ }_{f}=3.5 \mathrm{~m}$ the expected gain is also around 8 . It should be remembered that the Mushroom has $2 x$ the solid angle of LET with detectors on both sides so the real gain for most samples will be more like 16 . The overall gain though must take into account losses from the velocity selector which is shown in the next section. Taking these losses into account the overall gain of the Mushroom is closer to $\approx 10 \times$ LET. 


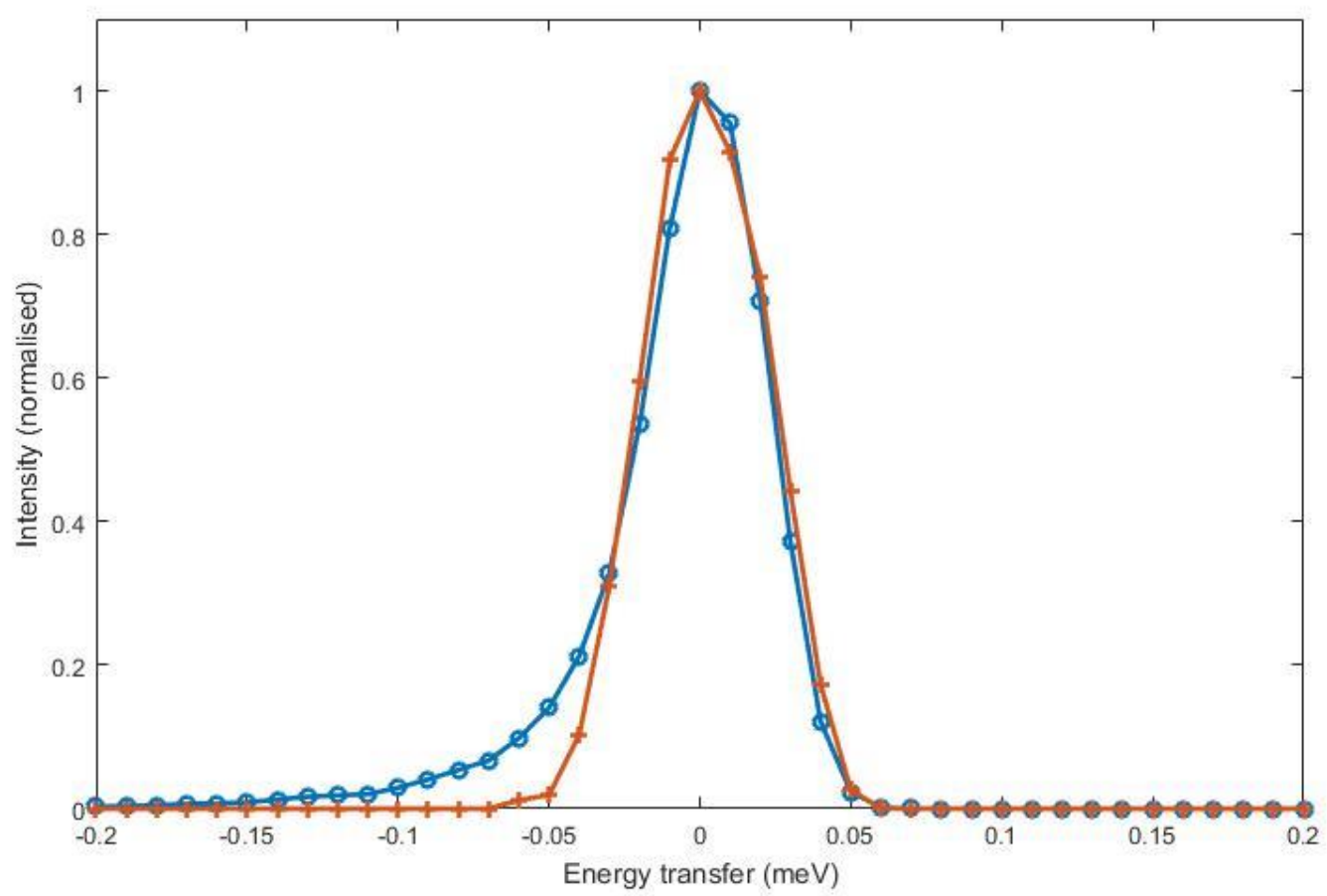

Figure 8. The elastic line for Mushroom (circles) and LET (crosses). The energy resolution of LET has been set equal to Mushroom and both have been normalised to a peak intensity of 1.

\subsection{Background and velocity selector}

Mushroom has been carefully designed such that the analysed neutrons are focussed through a small radial opening where it passes through a velocity selector. There are two reasons for this important design feature, the first being that passing the neutrons through a radial point enables the construction of a velocity selector as shown in figure 2 . This velocity selector enables the clean selection of either the PG002 or PG004 reflection by running the velocity selector at 30 or $60 \mathrm{~Hz}$ respectively. The velocity selector does not need to be phased to any timing signal and even the frequency does not need great accuracy so it is simple to run. The neutron absorbing blades of the selector have a $5 \mathrm{~mm}$ pitch at their centres and are $8 \mathrm{~cm}$ long and set at an angle of $14^{\circ}$ off vertical. On average the neutrons pass vertically down through the selector from the analyser but with a range of $\approx \pm 30^{\circ}$ off vertical for neutrons coming from the highest and lowest analyser angles. The off vertical angle lowers the vertical velocity component (increasing the effective vertical wavelength component) through the selector and reduces the probability of it being transmitted. The velocity selector was designed such that it is 'sloppy' enough to boost the transmission over the desired wavelength range but just 'tight' enough to stop the transmission of other orders. This can be seen in figure 9 which shows the transmission versus the vertical component wavelength for the velocity selector. The striped area represents the effective wavelength range from the Mushroom analyser showing that the transmission peaks at $\approx 90 \%$ and drops to around $60 \%$ at the extremes. The 
velocity selector has clear advantages over a Beryllium filter in that it can cleanly select PG002 or PG004 whereas the Beryllium filter can only select PG002. Also the velocity selector acts as both a high and low pass filter compared to the low pass Beryllium filter, thus reducing possible backgrounds.

The second important reason for this design is to minimise background and possible spurious signals. The main contribution to the background from PG crystals comes from thermal diffuse scattering (25). One way to reduce this effect is to cool the PG crystals as is done on the IRIS and OSIRIS spectrometers and in principle could also be done with the Mushroom analyser. Another way to reduce the diffuse scattering reaching the detectors is to minimise the solid angle view of the analyser from the detector which is precisely what the Mushroom geometry does very effectively. Each point on the detector can only see a very small section of the analyser looking through both the collimator blades and the radial ring. The collimation is not just spatial but also temporal as the velocity selector will reduce possible spurious signals from neutrons with the wrong final energy. Although this design does as much as possible to reduce backgrounds it should be noted that the Mushroom instrument like any indirect instrument will still not have backgrounds as low as DGS instruments like LET which have monochromatic incident beams and effects of scattering from analysing crystals.

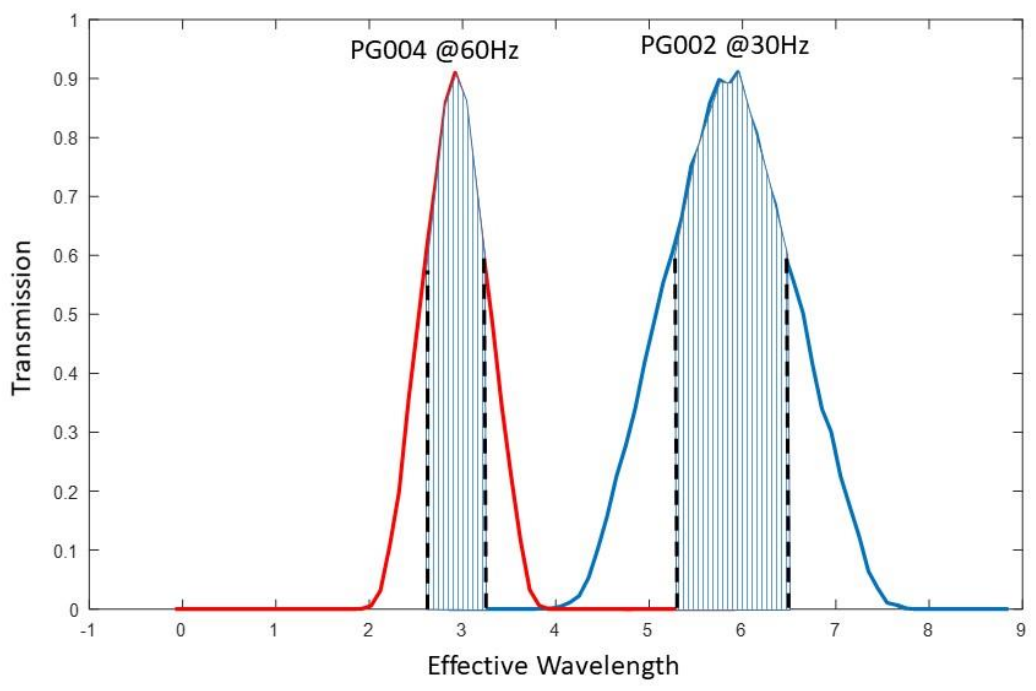

424

Figure 9. Transmission of the velocity selector versus the vertical component of the wavelength or 'effective' wavelength. The striped regions represent the effective wavelength range from the Mushroom analyser. To select the PG002 or PG004 reflection the velocity selector is run at a frequency of 30 or $60 \mathrm{~Hz}$ respectively.

\section{Summary and discussion}

This paper has presented a concept for an in-direct tof spectrometer called Mushroom which has a massive $2 \pi$ steradians of continuous position sensitive detector coverage, emulating 
some of its direct geometry counterparts such as IN5 or LET. It has advantages over its direct geometry cousins, such as being much more compact with the Mushroom having a radius of just $1.4 \mathrm{~m}$. It is also much cheaper as massive areas of ${ }^{3} \mathrm{He}$ position sensitive detectors are not needed. The Mushroom needs needs $1.7 \mathrm{~m}^{2}$ of PG crystal analyser to cover $\pi$ steradians of solid angle (similar to LET) costing $\$ 1.2 \mathrm{M}$ to $\$ 1.8 \mathrm{M}$ depending on whether $0.8^{\circ}$ or $0.5^{\circ}$ mosaic crystals are used respectively and another $\$ 0.5 \mathrm{M}$ for the detector array. For the same solid angle coverage a direct geometry instrument like LET needs around $40 \mathrm{~m}^{2}$ of ${ }^{3} \mathrm{He}$ detectors costing around $\$ 10 \mathrm{M} \mathrm{(27)} \mathrm{at} \mathrm{the} \mathrm{time} \mathrm{of} \mathrm{writing} \mathrm{(assuming} 2.5 \mathrm{~cm}$ diameter tubes with $6 \mathrm{~atm}$ ${ }^{3} \mathrm{He}$ pressure) and at least another $\$ 1 \mathrm{M}$ is needed for the very large detector tank. However, the main advantage is its much higher count rate for the same resolution. It was shown that the Mushroom will have about an order of magnitude higher count rate than LET, thus allowing much more rapid mapping of $S(Q, \omega)$ space for single crystals. This opens up the opportunity to do parametric HORACE scans or just to measure smaller crystals.

Minimisation of background was at the forefront of considerations when designing Mushroom, particularly from the thermal diffuse scattering of PG crystals. The focussing design of the analyser through a point at the velocity selector tightly collimates the beam both spatially and temporally to maximise background reduction. In addition this design enables the use of a mechanical velocity selector to easily select the PG order to use. Although it is envisaged that PG002 will be used predominantly, PG004 would be selected if a larger Q range is necessary although this comes at the price of worse energy and $Q$ resolution. This is in much the same way as you would increase $E_{i}$ on a direct geometry instrument to increase $Q$ range, also at the expense of energy and $Q$ resolution.

Mushroom has some limitations compared to a DGS. The mosaic spread of the PG crystals degrades the $Q$ resolution but the effect is minimal as large incoming beam divergences from the neutron guides, necessary to increase the count rate on low counting inelastic spectrometers, tend to dominate the $\mathrm{Q}$ resolution. Sample size will affect the energy resolution of the Mushroom, and therefore it is vital that beam slits are used just before the sample to define a beam size on sample. Mushroom will not have the flexibility of a DGS where one can choose any incident energy and resolution within the mechanical limits of the choppers, although the upshot of this is the simplicity of operation with just two modes to choose from (PG002 and PG004). If it was built on a reactor source or a long-pulse spallation source like the ESS then there is the possibility to use a pulse shaping chopper to vary the resolution of Mushroom. Although much effort has gone towards minimising Mushroom background it is never going to be as good as a direct geometry instrument like LET.

Finally there is no reason why Mushroom could not go onto a reactor source. One could employ choppers to pulse the beam and use it in a time of flight mode as in this paper, but a more efficient mode would probably use a PG monochromator. Just like a triple axis instrument the Mushroom analyser would rotate around the monochromator to scan through $E_{i}$ and hence the energy transfer. Colleagues at FRMII are studying this possibility at present (28). 
The author would like to thank Alex Buts for software help, Peter Galsworthy for the engineering figures and Russell Ewings for keeping the pressure on to finish this.

1. G. Ehlers, A. A. Podlesnyak, J. L. Niedziela, E. B. Iverson, and P. E. Sokol. 2011, Review of Scientific Instruments, Vol. 82085108.

2. J. Ollivier, H. Mutka and L. Didier. 2, 2010, Neutron news, Vol. 21.

3. R.I.Bewley, J.W.Taylor and S.M.Bennington. 1, 2011, Nuclear Instruments and Methods in Physics A, Vol. 637.

4. J.R.D.Copley, J.C.Cook. 2-3, 2003, Chemical Physics, Vol. 292.

5. Tobias Unruh, Jurgen Neuhaus, Winfried Petry. 3, 2007, Nuclear Instruments and Methods in Physics A, Vol. 580.

6. Kenji Nakajima, Seiko Ohira-Kawamura, Tatsuya Kikuchi, Mitsutaka Nakamura, Ryoichi Kajimoto, Yasuhiro Inamura, Nobuaki Takahashi, Kazuya Aizawa, Kentaro Suzuya, Kaoru Shibata, Takeshi Nakatani, Kazuhiko Soyama, Ryuji Maruyama, Hiromichi Tanak. SB028, 2011, J. Phys. Soc. Jpn, Vol. 80.

7. R.A. Ewings, A. Buts, M.D. Lee, J. van Duijn, I. Bustinduy, T.G. Perring. 2016, Nuclear Instruments and Methods in Physics Research A, Vol. 834.

8. R.I. Bewley, T. Guidi and S. Bennington. 1, 2009, Notiziario Neutroni e Luce di Sincrotrone, Vol. 14.

9. R. Bewley, R. Eccleston. 2002, Appl. Phys. A, Vol. 74.

10. The 3He Supply Problem. Kouzes, R. T. 2011, Technical Report 11-753, US Government Accountability.

11. $10 B$ multi-grid proportional gas counters for large area thermal neutron detectors. T. Bigault, J. Birch, J.C. Buffet, J. Correa, R. Hall-Wilton, L. Hultman, C. Hoglund, B. Guerard, A. Khaplanov, F. Piscitelli, P. Van Esch. 2012, Scientific reviews.

12. http://www.esss.se.

13. https://europeanspallationsource.se/instruments/cspec.

501

14. https://europeanspallationsource.se/instruments/t-rex.

15. J.Kulda, M.Kempa B.Janousova J.Saroun P.Flores M.Boehm F.Demmel. 2006, Physica B: Condensed Matter, Vols. 385-3862.

16. Kulda, J. 433, 2005, Nucl. Eng. Technol, Vol. 38.

17. Oleg Sobolev, Ron Hoffmann, HolgerGibhardt, Norbert Jünke, Andreas Knorr, Volker Meyer, Götz Eckold. 2015, Nuclear Instruments and Methods in Physics A, Vol. 772. research, Vol. 17. 
510 20. J.Zikovsky, O.Arnold J.C.Bilheux J.M.Borreguero A.Buts S.I.Campbell L.Chapon M.Doucet

511 N.Draper R.Ferraz Le M.A.Gigg V.E.Lynch A.Markvardsen D.J.Mikkelson R.L.Mikkelson R.Miller

512 K.Palmen P.Parker G.Passosa. 11, 2014, Nuclear Instruments and Methods in Physics Research A,

513 Vol. 764.

514 21. https://www.mantidproject.org/Main_Page.

515 22. F. Demmel, K. H. Andersen. 2008, Measurement Science and Technology, Vol. 19, p. 034021.

516 23. Keener, K. W. Herwig W. S. Appl. Phys. A, Vol. 74.

517 24. Panasonic, Highly Orientated Graphite catalog 2015/04.

518 25. C. Carlile, M.A. Adams. 4, 1992, Physica B, Vol. 182.

519 26. D. Martín, y Marero and D.Engberg. 1999, Physica B, Vol. 268.

520 27. Private communication.

521 28. Private communication with Robert Georgii,Ran Tang and Peter Böni. 